\title{
GENERACION Y DIBUJO DE ESCALERAS CON EL PROGRAMA CADET
}

\author{
(CAD OF STAIR CASES USING THE PROGRAM CADET)
}

\author{
Alfonso Recuero, Olga Río y José Pedro Gutiérrez, Drs. Ings. de Caminos \\ IETCC/CSIC
}

\section{RESUMEN}

En el presente trabajo se muestra un ejemplo de las posibilidades que ofrece el programa CADET para el dibujo de perspectivas de objetos tridimensionales. Para ello, se ha elegido el caso de dibujo de perspectivas de escaleras, con plantas de forma general.

Se incluye una descripción detallada para un ejemplo concreto, asi como, las perspectivas obtenidas para una variada gama de casos.

Se incluyen también, los listados de las subrutinas, en HP-BASIC, que permiten definir la planta de la escalera y generar los elementos planos utilizados en su discretización.

\section{SUMMARY}

This paper shows some of the possibilities offers by the program CADET to do perspective drawings of 3-D objects. Stair cases with any plant have been chosen as sample case.

A detailed description of every step needed in a particular case is included together with the perspective drawings obtained in several other cases.

The listings of the subroutines, written in HP-BASIC, which allow the definition of the plant and the automatic generation of the elements used to discretize the stair cases, are also included.

\section{INTRODUCCION}

Como ilustración de las posibilidades que ofrece el programa CADET para el dibujo de perspectivas de objetos tridimensionales con ordenadores personales, descrito de forma completa en [1], se muestra en este trabajo la forma de definir escaleras de planta cualquiera para el dibujo de perspectivas de las mismas.

Con tal fin, se describen minuciosamente los pasos seguidos con un ejemplo concreto, y se muestran además otros ejemplos que ponen de manifiesto las variadas posibilidades en la definición de este tipo de objetos.

Se incluye también el listado de la subrutina que permite definir y generar los elementos planos utilizados en su discretización, escrita en HP-BASIC.

\section{Definición de una escalera}

El programa permite generar los elementos de una escalera con una definición en planta muy general. Los peldaños deben tener una huella en forma de cuadrilátero o de triángúlo y, en el primer caso, el sentido de avance debe ser a través de dos lados opuestos del cuadrilátero. Esta condición obliga, en el caso de escaleras con descansillo cuadrilateral, en el que el avance se hace a través de dos lados contiguos del cuadrilátero, a dividir la escalera en una serie de tramos entre descansillos, de modo que en cada tramo se cumpla la condición antes citada.

Debe empezarse por definir la escalera en planta, generando una secuencia de vértices que contenga las proyecciones de las esquinas de los escalones, para lo cual puede utilizarse el procedimiento descrito en [3]. Las proyecciones de las esquinas de cada uno de los dos bordes de la escalera deberán estar situadas, consecutivamente, en la secuencia. El programa pedirá el primero y último vértices de los bordes interior y exterior. Ambos bordes deben tener igual número de puntos, pudiendo ser la numeración de los bordes as. ceiidente o descendente. En caso de existir escalones triangulares deberán también existir puntos sucesivos en el borde correspondiente con iguales coordenadas en planta. 
El programa considera como proyecciones de los bordes de los escalones las rectas que unen parejas de puntos que ocupan la misma posición en ambos bordes. Se presenta, entonces, en pantalla la planta de la escalera, pidiendo conformidad al operador.

Si la planta es correcta, el programa pedirá las coordenadas globales del origen local y las coordenadas de un punto del eje $X$ positivo local. Dado que se considera la escalera vertical, las coordenadas $Z$ de ambos puntos deberán coincidir.

A continuación el programa pedirá la altura de la escalera, si deben o no generarse las contrahuellas y si debe asignarse espesor a los escalones, así como el número del objeto en el que se considera incluida la escalera. Además, en el caso de que se asigne espesor a los escalones y se generen las contrahuellas preguntará si el tramo generado es el primer tramo de la escalera.

El programa genera tantos escalones completos como puntos tiene cada borde menos uno, empezando siempre por la contrahuella, si existe. Las contrahuellas serán siempre rectangulares, en tanto que la forma de las huellas dependerá de la definición en planta de la escalera. En caso de tener que asignar espesor a los escalones, el programa construirá, sobre huellas y contrahuellas, un prisma recto de altura igual al espesor asignado (ver Subrutina ESCALERA).

\section{Ejemplos de utilización}

Considérese, por ejemplo, la escalera de la que se muestra una perspectiva cónica en la figura 1 , la cual tiene una primera parte recta, una segunda parte semicircular y una tercera parte nuevamente recta, todas ellas seguidas sin descansillos intermedios.

Una forma de generarla por medio del programa CADET es la siguiente: Se define en primer lugar un bosquejo de la planta dando las coordenadas de los puntos 1 y 2 , a continuación el arco de circunferencia definido por los puntos 2 al 10, los puntos 11,12 y 13 mediante sus coordenadas, el arco de circunferencia definido por los puntos del 13 al 21 y, por último, el punto 22 mediante sus coordenadas. Con ello se obtendrá la secuencia de puntos que puede verse en la figura 2.

Seguidamente, se intercalan linealmente 2 puntos antes del 22, tres puntos antes del 13, tres puntos antes del 11 y dos puntos antes del 2. Con estas operaciones, la secuencia de puntos obtenida será la de la figura 3.

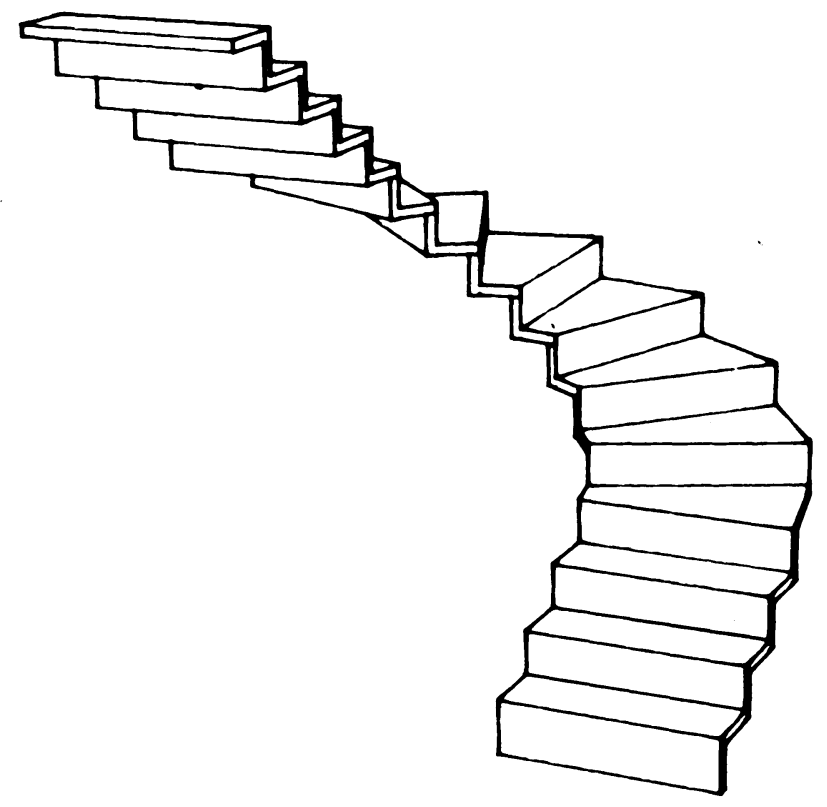

Fig. 1

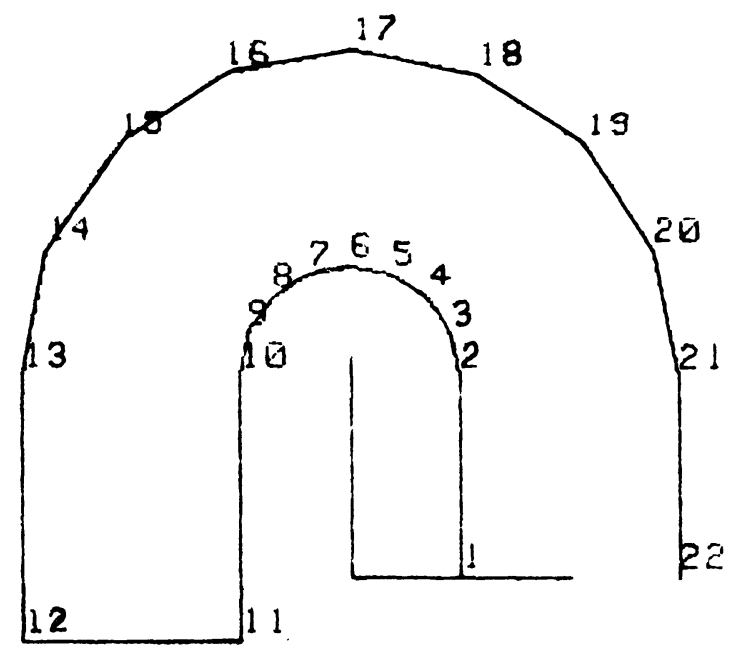

Fig. 2

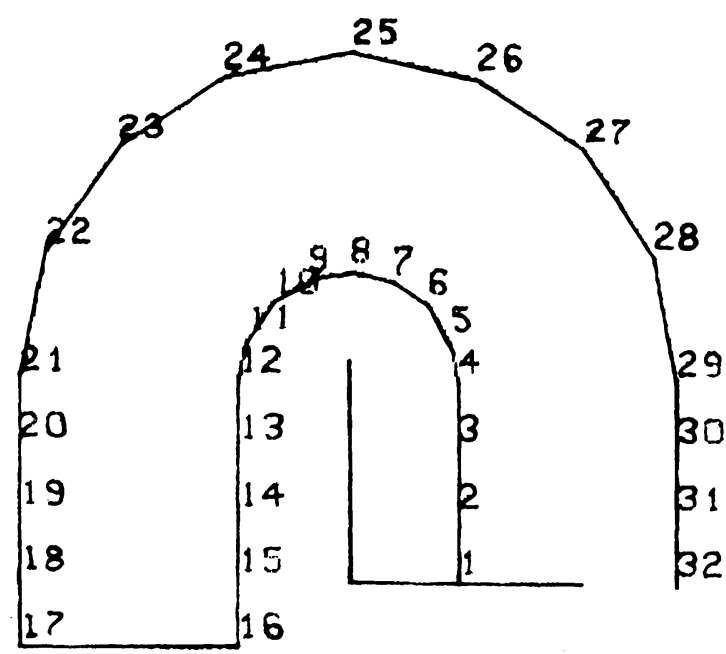

Fig. 3

http://informesdelaconstruccion.revistas.csic.es 


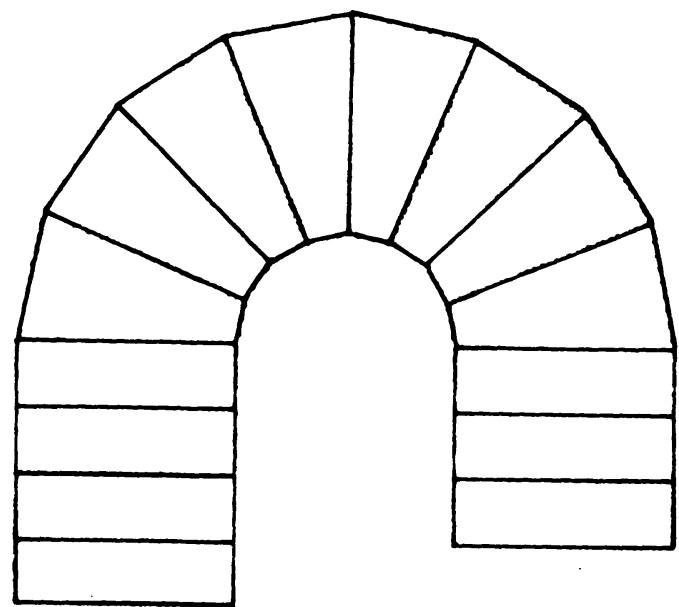

Fig. 4

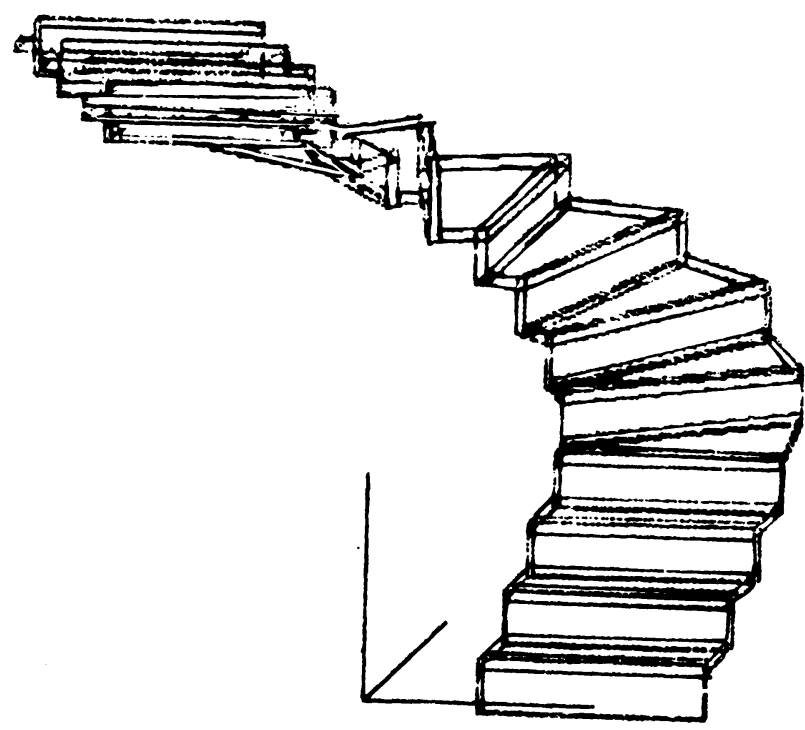

Fig. 5

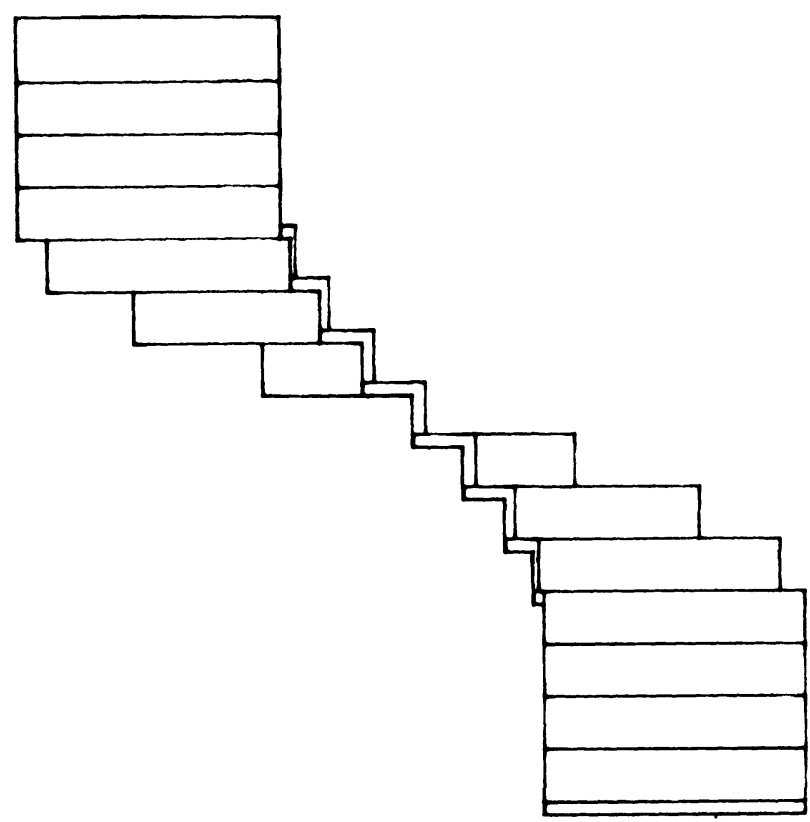

Fig. 6
A partir de esta secuencia puede ya describirse la planta de la escalera. Para ello se indicará que el borde interior está definido por los puntos 1 al 16 y el borde exterior por los puntos 32 al 17. Tras ello el programa mostrará la planta de la escalera tal como aparece en la figura 4.

Si el operador da la conformidad a esta planta, el programa pedirá las coordenadas globales del origen lo$\mathrm{cal}$, las coordenadas de un punto del eje $\mathrm{X}$ positivo lo$\mathrm{cal}$, la altura de la escalera, si deben o no generarse las contrahuellas de los escalones, el espesor que se desea asignar a los mismos, si se trata o no del primer tramo de escalera, y por último el número del objeto en que se considere incluida la misma. Una vez suministrados todos estos datos, el programa generará los elementos en que se discretiza la escalera, y presentará por pantalla una perspectiva caballera del objeto completo, incluida la escalera. En esta perspectiva figuran todas las aristas del objeto, sin determinar las partes vistas u ocultas. El aspecto de esta perspectiva puede verse en la figura 5 .

Si el operador da su conformidad a esta generación podrá seguir definiendo nuevos elementos o pasar a dibujar los ya generados. Como ya se ha indicado, en la figura 1, se muestra una perspectiva cónica de la escalera asi generada. Es posible obtener cualquier tipo de perspectiva axonométrica o cónica de la misma, así, en la figura 6 , se presenta un alzado de la escalera.

Es posible generar escaleras asignando un espesor 0 a los escalones, como es el caso de la figura 7.

Pueden también generarse escaleras sin contrahuellas. Así en las figuras 8,9 y 10 se muestran respectivamente la planta, el alzado y la perspectiva de una escalera de caracol. En este caso la escalera se ha completado con un tubo central, la barandilla y una plataforma de desembarco en el piso superior.

Como ya se ha indicado, en el caso de que existan descansillos cuadrilaterales en los que el sentido de avance se haga a través de dos lados contiguos, la escalera debe dividirse en un conjunto de tramos, en cada uno de los cuales se respete el sentido de avance indicado en el apartado anterior. Así, en la escalera de la figura 11, ha sido necesario generar 3 tramos: el primero incluye el primer tramo de escalones y el primer descansillo; el segundo tramo de escalones y el segundo descansillo; y el tercero el tercer tramo de escalones.

También deberán definirse en varios tramos las escalinatas de acceso a entradas principales con varios frentes de escalones, como la que muestra la figura 12. 


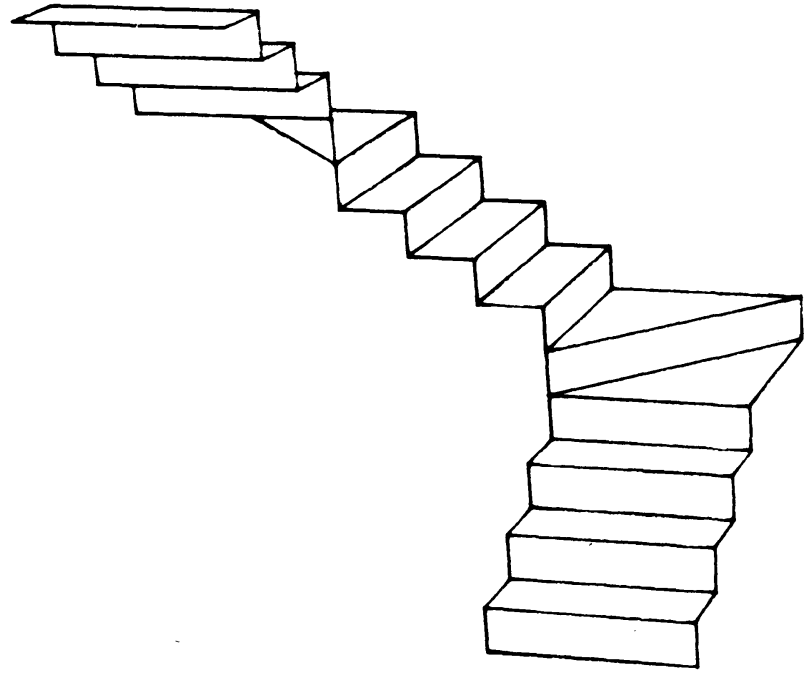

Fig. 7

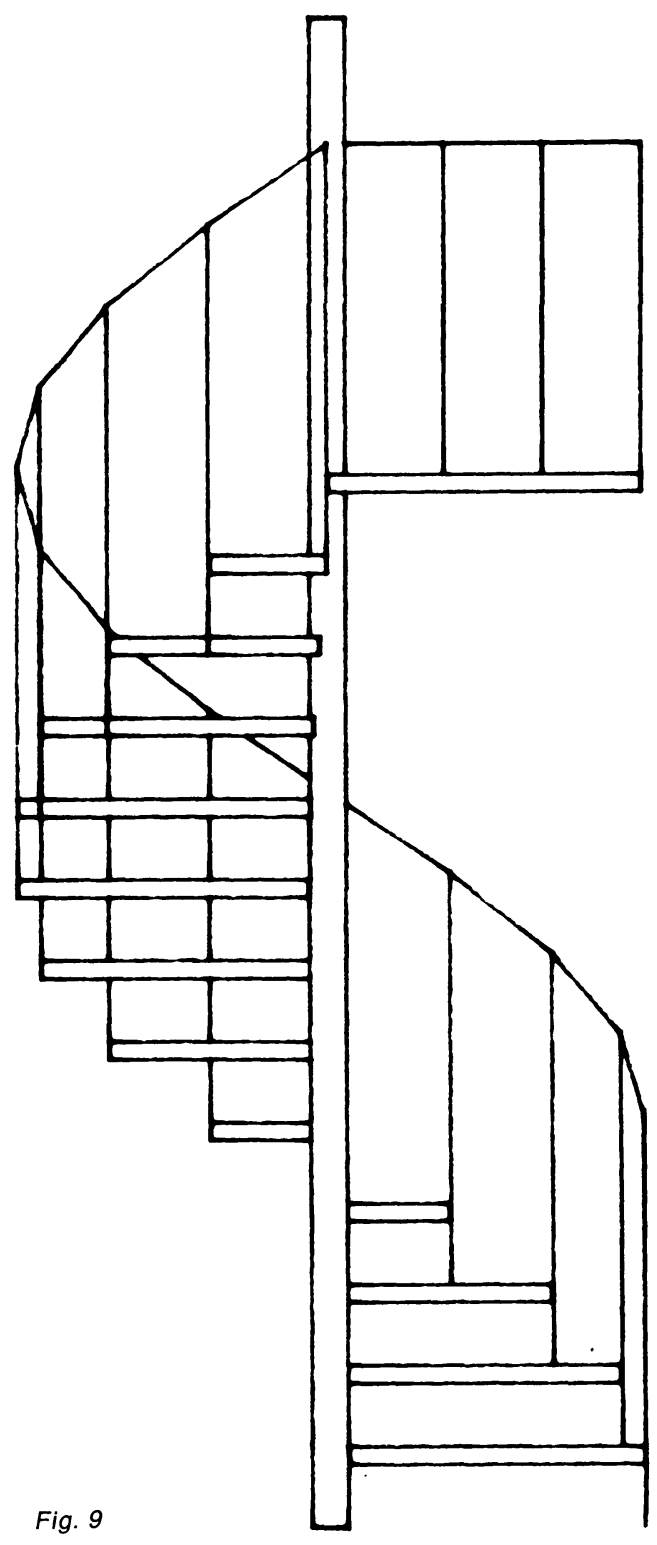

(c) Consejo Superior de Investigaciones Científicas Licencia Creative Commons 3.0 España (by-nc)
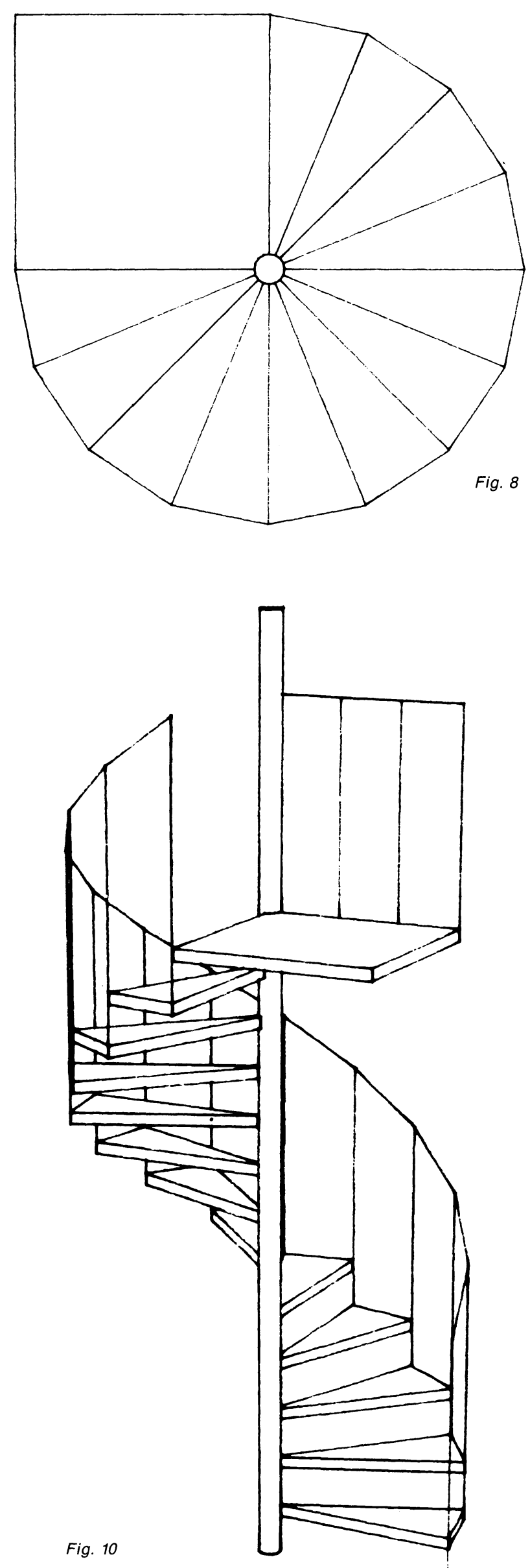

http://informesdelaconstruccion.revistas.csic.es 


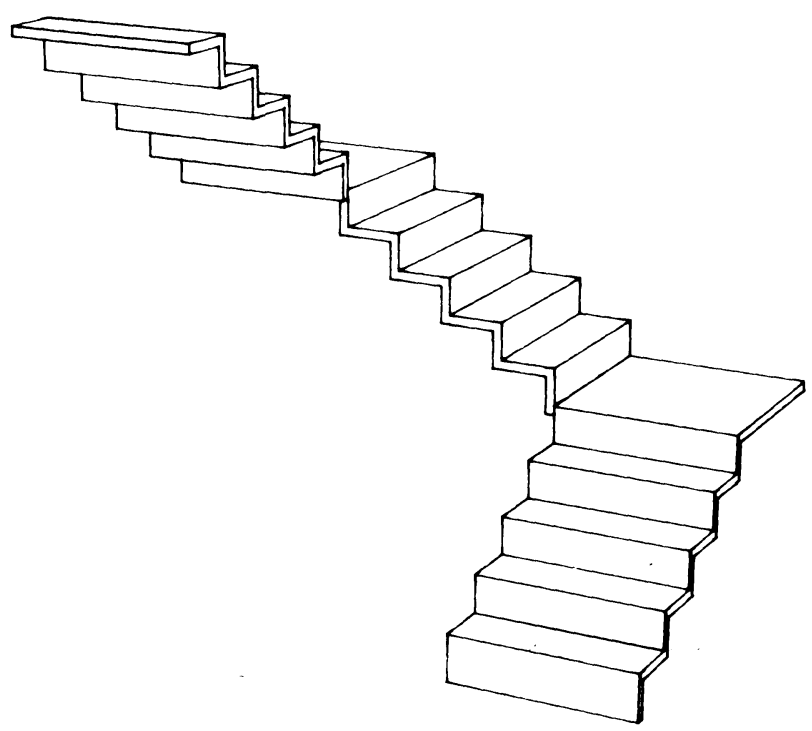

Fig. 11

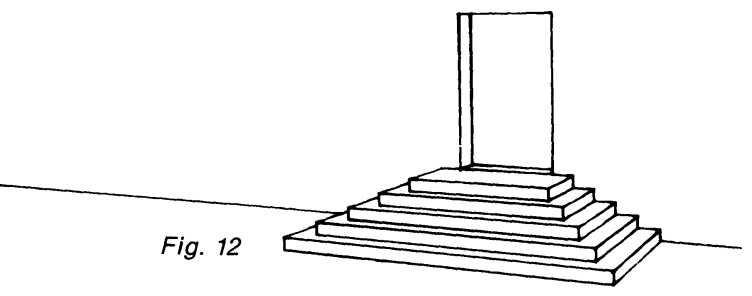

Como puede apreciarse en los tramos rectos de escalones con espesor, los bordes de los escalones presen- tan la superficie continua, en tanto que en los tramos curvos se marcan en el perfil cada uno de los escalones de forma independiente dado que los planos del perfil de huella y contrahuella no son coincidentes, ya que el espesor se ha asignado construyendo sobre huella y contrahuella un prisma recto de altura igual al espesor.

Por último puede señalarse que para construir elementos auxiliares, tales como: barandillas, paredes, tubos interiores, etc. puede utilizarse también la descripción en planta de la propia escalera que servirá para situar en el espacio, a la cota que corresponda, los puntos necesarios para definir dichos elementos auxiliares.

\section{Bibliografía}

1. Recuero, A.; Gutiérrez, J. P.; Rio, O!'Dibujo de perspectivas, asistido por computador, utilizando dispositivos trazadores gráficos". Monografía n. ${ }^{\circ} 390$ del Instituto Eduardo Torroja de la Construcción y del Cemento. Madrid, 1987 (en prensa).

2. Recuero, A.; Gutiérrez, J. P:‘Perspective drawing of three dimensional objects". Microsoftware for Engineers. Abril, 1987.

3. Recuero, A.; Río, O.; Gutiérrez, J. P:‘Generación de superficies curvas utilizando el programa CADET". Informes de la Construcción n. ${ }^{\circ} 387$. Enero-febrero de 1987.

4. Recuero, A.; Río, O.; Gutiérrez, J. P:‘'Dibujo de perspectivas de escaleras con ayuda de un computador". Montajes e Instalaciones n. ${ }^{\circ}$ 193. Marzo de 1987.

\section{Apendice}

Listado de la subrutina escalera

Las subrutinas llamadas por la subrutina escalera y que no figuran en este listado están contenidas en el listado completo del programa CADET que puede verse en (1).

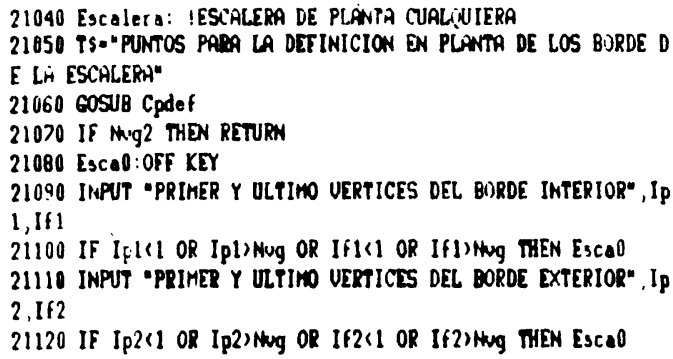

21130 IsI-Sen(IfI-Ipl)

21140 Is2=SQN(If2-Ip2)

21150 If Is $1 *(I f 1-I p 1)(1)] s 2 \star(I f 2-I p 2)$ OR Is $1=0$ THEN

21160 DISP "BORDES INCONSISTENTES PULSAR CONT PARA SEGUIR"

21170 PAUSE

21180 GiTO Escalor

21190 END If

21200 PRINT USING "Q , 80A"; "PLANTA DE LA ESCALERA"

21210 ECLEAR

21220 HUUE Xug(Ipl, 1),Xug(Ip1,2)

21230 FOR I-Ipl-IsI to IfI STEP IsI

21240 DRAW Xug $(1,1), \operatorname{Xug}(1,2)$

21250 NEXT I

21260 MOUE $X(\mathrm{~g}(\operatorname{Ip} 2,1), \mathrm{Xug}(\mathrm{Ip} 2,2)$

21270 FOR 1-Ip2+1.2 TO If2 STEP Is2

21280 DRAW $X u g(1,1), x \cup g(1,2)$

21290 NDXT I

$\left.21300 I=I \frac{1}{6}\right]$

$21310 \mathrm{~J}=1 \mathrm{p} 2$ 
21320 FI)R $K=$ Ipl TO IfI STEP ISI

21330 HOUE $X u g(1,1), X \cup g(1,2)$

21340 DRow $x \cup g(J, 1), x \cup g(J, 2)$

$21350 \mathrm{~J}-\mathrm{I} \cdot \mathrm{Is} 1$

$21300 \mathrm{~J}=\mathrm{J} \cdot \mathrm{J} 3 \mathrm{~g}$

21370 NEXT K

21381 OII KEY 5 LABEL "KS UALE" GOTO EscaU1

21390 ON KEY 8 LABEL. "K8 NO UALE" GOTO Escalere

21400 GUTO Espere

21410 EsCaO1:0FF KEY

21420 Escal : INPUT "COORDENARAS DEL. ORIGEN LOCAL", XaL $(*)$

21430 INPUT -COURDOADAS DE UN PUNTO DEL EJE $X$ LOCAL POSITIU

$0^{\circ}, x_{0}(\$)$

n14i0 If $\left(X_{a a}(1)=X_{0}(1)\right.$ AND $\left.X_{a a}(2)=X_{a}(2)\right)$ OR Xae $(3)(1) X_{0}(3)$ TH

DN Escal

$21450[!(3,1)=0$

21460 Ej $(3,2)=0$

21470 E $(3,3) \cdot 1$

21480 COSUB E

21490 ESCO2: INPUT " GLTURA TOTAL", Hz

21500 If $\mathrm{Hz}=\mathrm{O}$ THEN EsC 2

21510 INPUT "CONTRAHUELCAS (O-N) l-SI)", Ch

21520 INPUT "ESPESOR DE LOS ESCALONES",

$21521 \mathrm{Pt}=1$

21522 If Ch AND LO THEN INPUT "ES EL PRIMER TRAHO? ONNO I=S

$I^{\circ}, \mathrm{Pt}$

2153000508 objeto

$21540 \mathrm{Nt} 1-1 \mathrm{~s}) *(\mathrm{IfI}-\mathrm{IpI})$

$21550 \mathrm{Hzl} \cdot \mathrm{Hz} / \mathrm{Nt} 1$

21500 REM GENERACION DE LOS UERTICES

$21570 \mathrm{I}-\mathrm{IpI}$

$21580 \mathrm{~J}=\mathrm{I} p 2$

21590 Nuantitu

$21600 \mathrm{~Hz} 2=\mathrm{O}$

21610 FÜR Il=IpI $m$ IfI STEP Is]

21620 FOR $\mathrm{Jl}-1$ TO 3

21630 If $\mathrm{Jl}=2$ THEN $\mathrm{H}_{2} 2-\mathrm{Hz}_{2} 2-6$

21640 If $\mathrm{Jl}=3$ THEN Hz2-Hz2 $+\mathrm{Lo} \cdot \mathrm{Hzl}$

$21650 \mathrm{Nu}=\mathrm{Nu}+2$

21660 FOR K-1 TO

$216>0 X(N u-1, K)-X_{A A}(K)+X u g(I, 1) \star E j(1, K)+X u g(I, 2) \notin E j(2, K)+H z 2$

$\pm E \mathrm{j}(3, \mathrm{~K})$

$21680 X(N u, K)=X_{a d}(K)+X u g(J, 1) \pm E j(1, K)+X u g(J, 2) \neq E j(2, X)+H z 2+E$

$\jmath(3, K)$

21690 NEXT $K$

21700 NEXT J1

21701 If I!) IPI AND Ch AND Lo THEN

21702 FOR Jl-N/W-5 TO $\mathrm{Nu}-4$

21703 FOR $K=1$ TO 3

$21704 \times x(K)=x(J 1, K)-x(J)-2, K)$

21705 NEXT $K$

21706 CALL Normal $\left(X_{x}(1), x_{x}(2), X_{x}(3), L\right)$

21707 FOR $X=1$ TO
$21708 \times(\mathrm{J} 1, K)=X(\mathrm{Jl}, K)+X \times(K)+L_{0} / 20$

21709 NEXT $K$

21710 NEXT JI

21711 END IF

$21713[-[+|s|$

$21720 \mathrm{~J} \cdot \mathrm{J} \cdot \mathrm{Is} 2$

21730 NDXT II

$21740 \mathrm{Nu}-\mathrm{Nu}-4$

21750 REM GENEDACION DE ELEMENTOS

21760 FOR IOSC=1 TO NTI

21770 If Ch THEN ICONTRAWUELLA

21780 6OSUB Prepare

$21790 \mathrm{Nab}(\mathrm{JO}+1)=\mathrm{Nab}(\mathrm{JO})+4$

21800 Dp $(K O)=N$ kes 3

21810 Dp $(K 0 \cdot 1)=-k_{0}+4$

21820 If 12:1 AND Pt THEN

21830 Dp $(K O)=K_{\text {twa }} 1$

21840 Dp (KO+1)-Hua+2

21850 DND If

21860 Op $(K O+2)-K_{1}+6$

21870 Dp $(K O+3)-N u a+5$

21880 GOSUB Elomento

21890 If Le THaN GOSUB Prismagen

21900 END If

21910 REM HUELLA

21920 605UB Prapara

$21930 \mathrm{Nab}(\mathrm{JO}+1)-\mathrm{Nab}(\mathrm{JO})+4$

21940 Dp (KO) -Neta+5

$21950 \mathrm{Dp}(\mathrm{KO}+1)-\mathrm{Kus}_{\mathrm{a}}+6$

21960 Dp $(K O \cdot 2)=$ Hua +8

$21970 \mathrm{Dp}(\mathrm{KO} \cdot 3)=\mathrm{Nun \cdot})$

21980 FOR $X=1$ TO 3

$21990 X X(K)-X($ Nue+7,,$K)-X($ Nua $\cdot 5, K)$

22000 MEXT $X$

22010 CALL Normal $\left(X_{x}(1), X_{x}(2), x_{x}(3), L\right)$

22020 If L $=0$ THEN Mab $(J 0 \cdot 1)-\mathrm{Hab}(\mathrm{J} O)+3$

22030 FOR $x=1$ TO 3

$22040 X \times(K)=x($ Nua $8, K)-X($ Nua 6,6,

22050 NDXT $X$

22060 CALL Normal $\left(X_{x}(1) ; X_{x}(2), X_{x}(3), R\right)$

22070 If $R=0$ THEN

22080 Dp $\left(\mathrm{K}_{0} \cdot 2\right)=\mathrm{Nua}+7$

$22090 \mathrm{Mab}(\mathrm{JO}+1)=\mathrm{Kab}(\mathrm{JO})+3$

22100 ENC If

22101 COSUB Aroas

22102 If $51-0$ THON Nepollep-1

22110 If S1 THEN GOSUB Elomento

22120 If Lo AND S1 NHEN COSUB Prismagen

22130 Nue-Nua+6

22140 NEXT lesc

22150 605JB Presenta

22160 PETURN 